\title{
Molecular analysis of Gpdh null mutations that arose in mutation accumulation experiments in Drosophila melanogaster
}

\author{
YUMI YAMAGUCHI†, TOSHIYUKI S. TAKANO†, TSUNEYUKI YAMAZAKI \& KO \\ HARADA* \\ Department of Biology, Faculty of Science, Kyushu University 33, Fukuoka 812, Japan
}

\begin{abstract}
In order to clarify the cause of null mutations in enzyme loci, the molecular structure of six null mutations in the Gpdh locus (encoding $\alpha \mathrm{GPOH}: \alpha$ glycerol-3-phosphate dehydrogenase $\left(\mathrm{NAD}^{+}\right)$, E.C. 1.1.1.8; map position at 2-17.8) that arose in mutation accumulation experiments was examined. A restriction map analysis showed that five of the mutations are insertional mutations whereas the sixth is a deletion. The $G p d h$ regions of these null mutations were then cloned and sequenced. The inserted DNA fragments are all internally deleted $P$ elements measuring $1.1 \mathrm{~kb}$ in length. Two are a KP element and two others are a HP element. All the insertions occur in the region near the initiation signal of transcription. The deletion encompasses the seventh and eighth exons over a length of $1.1 \mathrm{~kb}$. These results therefore indicate that the null mutation rate at the $G p d h$ locus is largely influenced by $P$ elements.
\end{abstract}

Keywords: Drosophila melanogaster, $G p d h$ gene, $H P$ elements, $K P$ elements, null mutations, $P$ elements.

\section{Introduction}

In previous mutation accumulation experiments, Mukai \& Cockerham (1977) estimated the spontaneous mutation rates to be $1.81 \times 10^{-6}$ for band morph and $1.03 \times 10^{-5}$ for null mutations at five enzyme loci ( $A d h, G p d h, M d h, H e x-C$ and $A m y$ ) on the second chromosome. After additional generations of mutation accumulation the band morph and null mutation rates were estimated to be $1.28 \times 10^{-6}$ and $3.86 \times 10^{-6}$ for a total of 3111598 allele generations (Voelker et al., 1980b). This is one of the most extensive studies ever carried out; however, it was suggested that these rates may be overestimated because many chromosome aberrations were found in these lines and the lines were thus suspected to carry mutator factors (Yamaguchi \& Mukai, 1976). It was shown that the mutation accumulation lines (AW and $\mathrm{JH}$ ) carried $l$ and hobo elements (but not $P$ elements) and that the transposition of these elements occurred rather frequently during the accumulation of mutations (Harada et al., 1990). To obtain more reliable mutation rates, a new series of experiments was conducted (Harada et al.,

*Correspondence.

†Present address: National Institute of Genetics, Mishima 411, Japan.
1993). In these experiments very old laboratory stocks (KA and KR series) or chromosomes with a native genetic background ( $\mathrm{KC}$ series) were used to minimize the influence of movable genetic elements. After a total of 1678388 allele generations of mutation accumulation, 44 null mutations were found at seven enzyme loci but no band morph mutations. Compared with previous experiments no statistically significant difference was detected for the band morph mutation rates. However, a significantly higher rate was observed for the null mutations at the $G p d h$ locus ( 25 null mutations among a total of 239533 allele generations (Harada et $a l ., 1993)$. To determine the cause of these null mutations and to assess the process of mutation accumulation, we investigated the molecular structure of six null mutations detected at the Gpdh locus.

\section{Materials and methods}

\section{Mutation accumulation lines}

Two series of mutation accumulation experiments, KA and $\mathrm{KR}$, were started in April 1982. Two independent lethal second chromosomes, $l(\mathrm{KA})$ and $l(\mathrm{KR})$, were sampled from the Kaduna laboratory stock (Daly \& Clarke, 1981). They were balanced with a laboratory balancer chromosome, $\ln (2 L R) S M I$ (abbreviated as 
Cy). Spontaneous mutations were accumulated on these chromosomes using the method described by Mukai \& Cockerham (1977). A similar experiment using a stem second chromosome, $l(\mathrm{KC})$, sampled from a natural population at Katsunuma, Japan was conducted in April 1984 (KC series). In the KC experiment, to reduce $\mathrm{P}-\mathrm{M}$ type hybrid dysgenesis a balancer stock, $\ln (2 L R) S M 1 / \ln (2 L R) b w^{V I}$ (abbreviated as $C y /$ $P m$ ) whose genetic background was substituted by that of an isofemale line from the Katsunuma population was used to expand the chromosome. Single-pair matings were used for accumulating mutations in these lines. Experimental crosses were performed at $25^{\circ} \mathrm{C}$. Screening for the electrophoretic variants was carried out periodically on the heterozygotes between the experimental lines and a tester strain that has normal and different allozyme types (Harada et al., 1993). The mutation accumulation lines were maintained in mass at $18^{\circ} \mathrm{C}$ after the final screening in November 1987.

\section{Establishment of $\mathrm{Cy}$ or lethal deficiency lines}

Males from the Gpdh null lines were crossed with $C y /$ $D f(2 L) G p d h A$ females. $D f(2 L) G p d h A$ is a deletion of the entire $G p d h$ region and is marked by a dominant marker $L$ (lobe eye) which reduces the eye size (Lindsey \& Zimm, 1992). In the next generation, $C y^{*} /$ $D f(2 L) G p d h A$ or $l^{*} / D f(2 L) G p d h A$ adults were collected. Males and females of the same genotype were crossed to establish the null-deficiency lines. Here, $C y^{*}$ or $l^{*}$ indicate $C y$ or lethal chromosomes, respectively, in which mutations have been accumulated. Six null lines, KA315l, KA699l, KR726 Cy, KR877l, KC198l and $\mathrm{KC} 303 l$ were established. Here, $l$ and $C y$ indicate that the mutation occurred in the lethal and $C y$ chromosomes, respectively. To construct a restriction map of the Gpdh region and to control the enzyme activity assay (described below), several non-null lines were chosen and either $C y$-deficiency or lethal-deficiency lines were established in the same way as in nulldeficiency lines (control lines). Cy/Df(2L)GpdhA was obtained from the Bowling Green Drosophila stock centre.

\section{Restriction map analysis}

The Gpdh region was examined by a Southern blot analysis of the null lines. Restriction maps were constructed for $C y, l(\mathrm{KA}), l(\mathrm{KR})$ and $l(\mathrm{KC})$ chromosomes using the control lines. Plasmids pG8S1 and pG9E (Takano et al., 1989) (Fig. 1) were used as probes. These probes cover a $26 \mathrm{~kb}$ region which includes the entire Gpdh coding region. The following four restriction enzymes were used in the analysis: HindIII, XhoI, $X b a \mathrm{I}$ and $\mathrm{SacI}$.

\section{Cloning and sequencing $\mathrm{Gpdh}$ null alleles}

After complete digestion by SalI (KA315l, KA699l, KR726 Cy and KR877l) or partial digestion by $M b o$ I (KC198l and $\mathrm{KC} 303 l$ ) genomic libraries were constructed and cloned into a $\lambda$ DASHII vector. Genomic clones including the Gpdh regions were isolated by plaque hybridization following standard procedures (Sambrook et al., 1989) using the plasmids pG8S1 and pG9E as probes. Each clone was then further subcloned into the plasmid vector Bluescript M13 using the appropriate restriction enzymes as shown in Fig. 1. Those subclones containing the regions of inser-

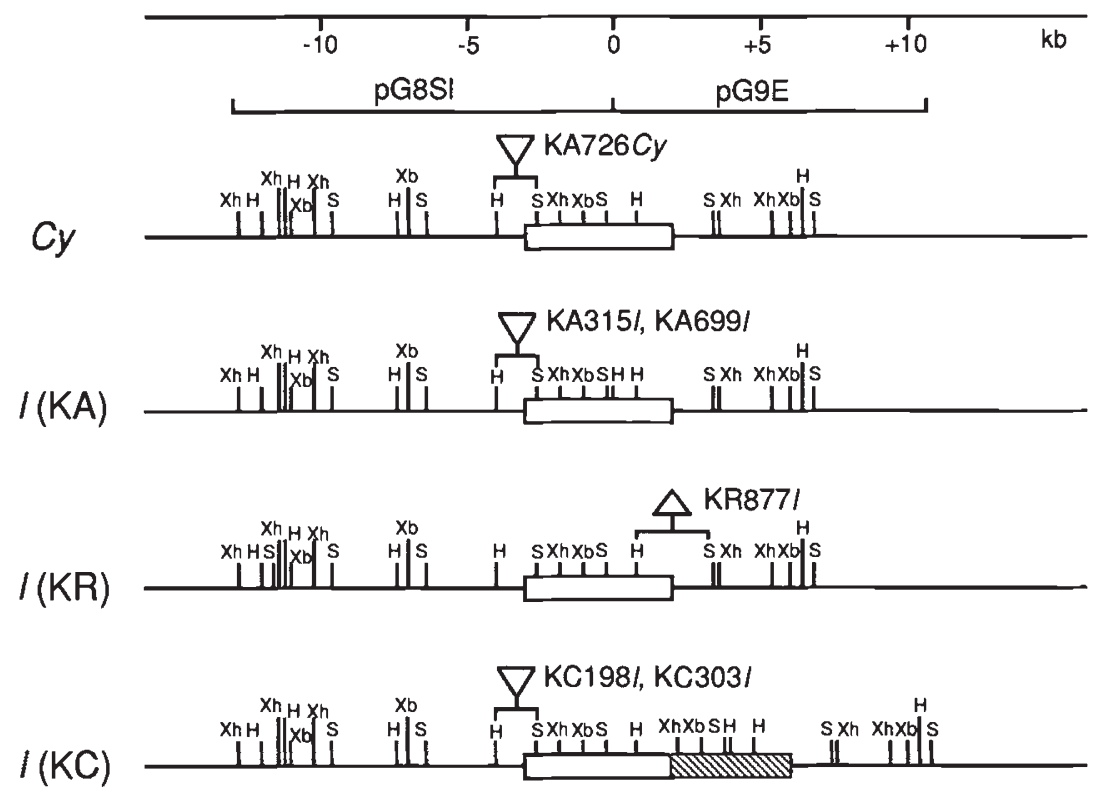

Fig. 1 Restriction map of the Gpdh region. The open box represents the transcriptional unit and its direction is left to right. The shaded box represents the partially duplicated fragment of the Gpdh gene (Koga et al., 1988). The restriction sites are indicated above the bar $(\mathrm{H}=$ HindIII, $\mathrm{Xh}=X h o \mathrm{I}$, $\mathrm{Xb}=X b a \mathrm{I}$ and $\mathrm{S}=S a c \mathrm{I})$. The two probes used in this experiment (pG8Sl and $\mathrm{pG} 9 \mathrm{E}$ ) are indicated above the map. Insertions and a deletion are indicated by triangles pointing towards or away from the map, respectively. The coordinates on the scale follow those of Bewley et al. (1989). 
tions or the deletion were isolated and sequenced. The synthetic oligonucleotides used as sequencing primers are 5'-AGGCGTTATACAGTACT-3', 5'-ATGTGTGGAGTGCAAAC-3'， 5'-TTGTCGATGGACTCGGT- ${ }^{\prime}$ and $5^{\prime}$-TCGTTGATAATCTCCGT-3' for the insertion mutations and 5'-ATGCTAAGTTGATGCAC- $3^{\prime}$ for the deletion mutation. A total of about 650 base pairs was sequenced for the regions containing the insertions and about 300 base pairs were sequenced for the region of the deletion (Fig. 1). Both strands of the cloned regions of the insertion mutations and a single strand for the deletion mutation were sequenced by the dideoxyribonucleotide chain termination method (Sanger et al., 1977). The precise position of the internal deletions of the defective $P$ elements was determined by sequencing using the $P$ element primers, P4 (5'-ACTGAAGAATGGTGGAC$\left.3^{\prime}\right)$ and P11 (5'-TTGCAATAAGTGCGAGT- $\left.3^{\prime}\right)$. P4 corresponds to the position 651 to 667 and P11 corresponds to the position 2850 to 2834 in the coordinate of $\mathrm{p} \pi 25.1$ element (O'Hare \& Rubin, 1983).

\section{Measurement of the specific activity of $\alpha$ GPDH}

All experimental flies were reared on a normal corn meal-molasses-yeast medium with four replicated vials. Five pairs were used as parents per vial and were discarded after 4 days. Emerging flies were aged for 4 days and from each vial two samples of five male flies were collected. The flies were homogenized in $0.2 \mathrm{~mL}$ of glycine- $\mathrm{NaOH}$ buffer ( $\mathrm{pH} 9.5$ ). After centrifugation, $25 \mu \mathrm{L}$ of supernatant was mixed with $2.4 \mathrm{~mL}$ of glycine- $\mathrm{NaOH}$ buffer, $50 \mu \mathrm{L}$ of $757 \mathrm{~mm}$ glycerophosphate and $50 \mu \mathrm{L}$ of $227 \mathrm{~mm} \mathrm{NAD}^{+}$. Changes in the optical density were measured with a spectrophotometer at the wave length of $340 \mathrm{~nm}$ after an interval of $30 \mathrm{~s}$ for $3 \mathrm{~min}$. Then, $50 \mu \mathrm{L}$ of supernatant was used to measure the amount of total soluble protein by the Lowry method (Lowry et al., 1951). The unit of specific activity was defined as the amount of enzyme activity that consumes one $\mu$ mole of coenzyme $\mathrm{NAD}^{+}$per $1 \mathrm{mg}$ of soluble protein per minute.

\section{Results}

\section{Restriction map analysis}

The restriction maps for each original chromosome were constructed using several control lines that were sampled from non-null lines in each experimental series. The $l(\mathrm{KC})$ chromosome has a partially duplicated Gpdh gene which is the same as that described by Koga et al. (1988) and Takano et al. (1989). For each original chromosome no restriction map polymorph- ism was detected among the control lines. The restriction maps of the null lines were compared with that of the control lines. Of the six null mutants, insertions were found in five (KA315l, KA699l, KR726Cy, $\mathrm{KC} 198 l$ and $\mathrm{KC} 303 l$ ) and a deletion was detected in the sixth mutant, KR877l (Fig. 1). The lengths of the inserted fragments are all about $1.1 \mathrm{~kb}$. All insertions were located between a HindIII site and a SacI site near the initiation signal of transcription. Within each insertion one or two HindIII sites and one XhoI site occur. The deletion, $1.1 \mathrm{~kb}$ long, occurs between the HindIII and SacI sites at the $3^{\prime}$ end of the transcription unit.

\section{Cloning and sequencing}

The regions including all insertions or the deletion of the six Gpdh clones were sequenced. The deletion mutant (KR877l) lacks a region of 1122 bp long that includes the entire seventh exon and most of the eighth exon. The breakpoints of the deletion are at positions 3785 and 4908 in the coordinate of Bewley et al. (1989) (Fig. 2 ). All the inserted fragments are internally deleted $P$ elements which measure 1068 to $1154 \mathrm{bp}$ in length. Their 8 bp target sites of insertion are shown in Fig. 3 . KA315l and KA699 $l$ are insertions at the same site (13 bp upstream from the initiation signal of transcription) in different orientations. The $P$ element of KA699 $l$ is inserted with its $3^{\prime}$ end to the left and in orientation opposite to the direction of transcription. KR726Cy has an insertion at 121 bp downstream from the initiation signal of transcription and 29 bp upstream from the initiation codon. $\mathrm{KC} 198 \mathrm{l}$ has an insertion at $24 \mathrm{bp}$ upstream from the initiation signal of transcription. $\mathrm{KC} 303 \mathrm{l}$ has an insertion at $13 \mathrm{bp}$ downstream from the initiation signal of transcription and $137 \mathrm{bp}$ upstream from the initiation codon and this $P$ element is inserted in orientation opposite to the direction of transcription. Breakpoints of the $P$ elements are shown in Fig. 4. Breakpoints of the $P$ element in KA699 $l$ and $\mathrm{KC} 303 l$ are the same as that of the $K P$ element (Black et al., 1987). Breakpoints of the $P$ elements in $\mathrm{KR} 726 C y$ and KA315l are also the same. This element is the common element HP (O'Hare et al., 1992). All the $P$ element insertions occur between the TATA box and the initiation codon. One polymorphic site was reported in the $K P$ element at position 32 where nucleotide $\mathrm{A}$ in $\mathrm{p} \pi 25.1$ is replaced with $\mathrm{T}$ (O'Hare $e t$ al., 1992). Another polymorphic site was reported in the $H P$ element at position 33 where $\mathrm{A}$ in $\mathrm{p} \pi 25.1$ is replaced with T (O'Hare et al., 1992). The $K P$ and the $H P$ elements found in our experiments also share the same polymorphic sites. In addition, the breakpoint on the left side of the internally deleted $P$ element in $\mathrm{KC} 198 l$ is the same as that of the KP element. Thus, 


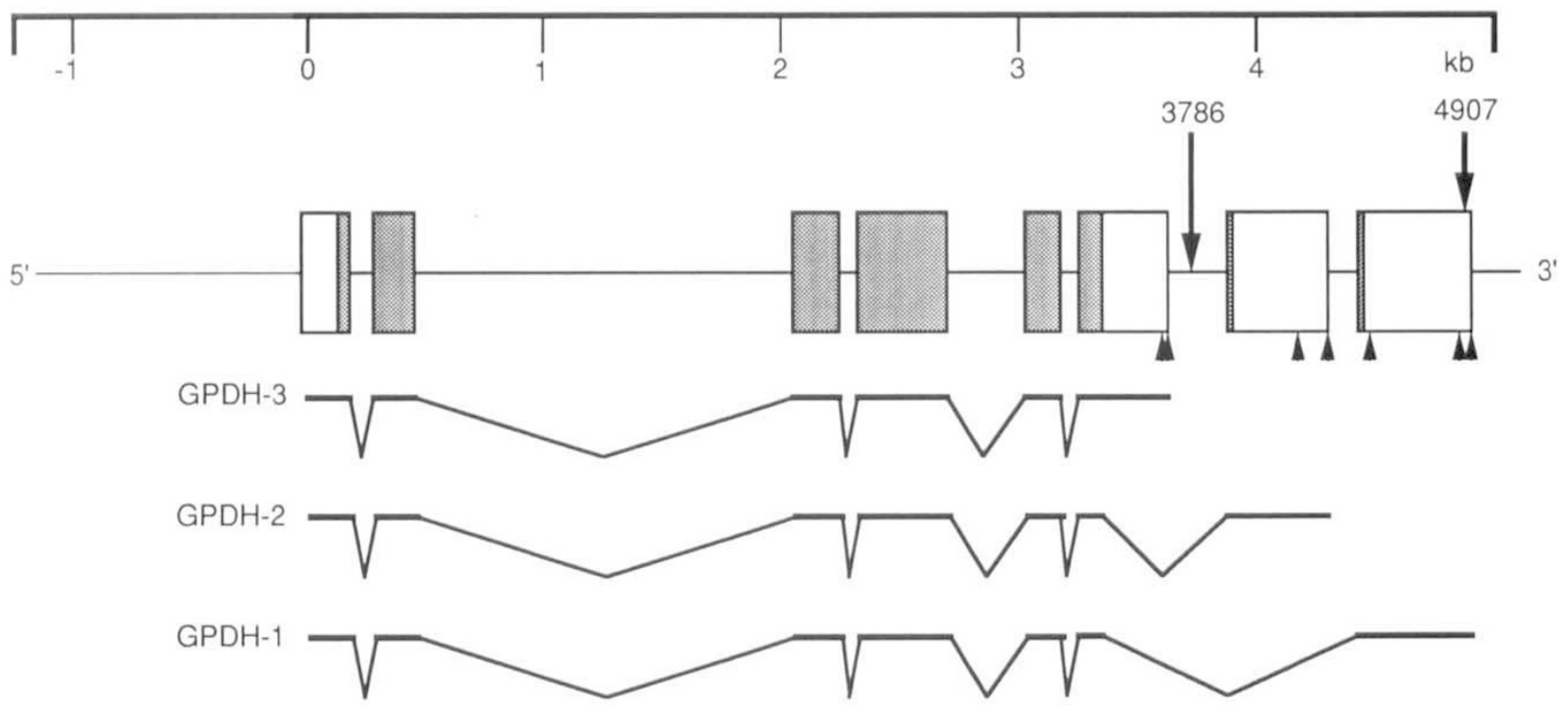

Fig. 2 Molecular structure of the $G p d h$ gene and the breakpoints of the deletional mutant (KR877l). The fine structure of the Gpdh gene is shown (Bewley et al., 1989) where the boxes indicate exons. The shaded and open boxes indicate the coding and nontranslated regions, respectively. The positions of the potential polyadenylation signals are shown by arrowheads. The two vertical arrows with numbers showing nucleotide positions indicate the breakpoints of the deletional mutant (KR877l). Three transcripts from the Gpdh locus, designated as GPDH-1, -2 and -3 are shown. Bewley et al. (1989) reported only the 3'-end of GPDH-2 but a complete structure of GPDH-2 is illustrated following their suggestion.

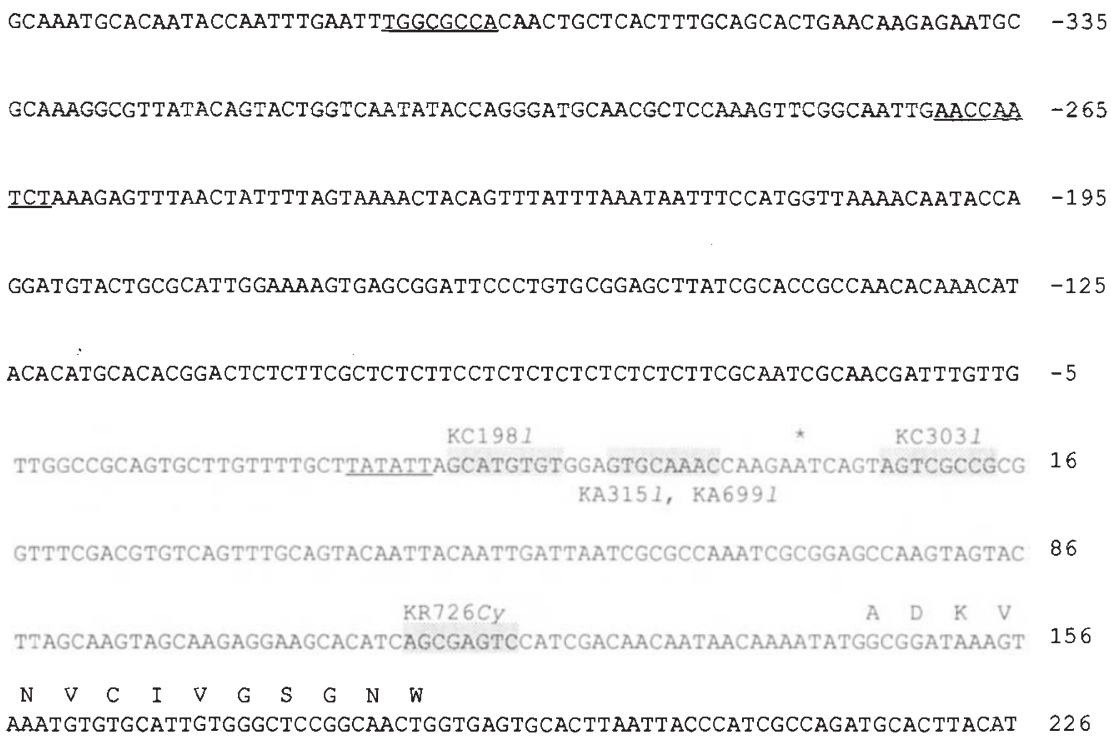

Fig. 3 Target sites of $P$ element insertions. The shaded 8 bp sequences show the duplicated target sequences of $P$ element insertions. The asterisk represents the initiation signal of the transcription. The initiation codon is located at the nucleotide position 143 . The TATA box, CAAT box and cAMP response element (CRE) homology site are all underlined. this element may have arisen by a further deletion of the $K P$ element.

\section{Measurement of the specific activity of $\alpha G P D H$}

The enzyme activities of the mutant lines and the control lines are shown graphically in Fig. 5 . $\mathrm{KR} 877 C y, \mathrm{KA} 382 l, \mathrm{KR} 209 l$ and $\mathrm{KC} 38 l$ are the control lines for the activity assay for $C y, l(\mathrm{KA}), l(\mathrm{KR})$ and $l(\mathrm{KC})$ chromosomes, respectively. No activity was detected in KR726Cy. KA315l and KA699l express partial activity but there is no significant difference between them $(34$ per cent of the activity of the control line, KA382l). KR877l has partial activity but at a very low level compared to the control line (13 per cent of the activity of the control line, KR209l). KC198l and KC303l have partial activity but there is a significant difference between them $(62$ per cent and 24 per cent of the activity of the control line, $\mathrm{KC} 38 \mathrm{l}$, respectively). Although their allozyme mobilities are the same, there 
Fig. 4 Molecular structure of the inserted defective $P$ elements. The numbered and shaded boxes represent the exons and introns, respectively. Restriction sites are indicated above the boxes $(\mathrm{H}=$ HindIII, $\mathrm{Xh}=X h o \mathrm{I}$, $\mathrm{E}=E c o \mathrm{RI}$ and $\mathrm{Sl}=S a l \mathrm{I})$. The thick horizontal lines represent regions of inserted fragments homologous to a complete $P$ element sequence. The numbers beside the lines indicate the breakpoints of defective $P$ elements.
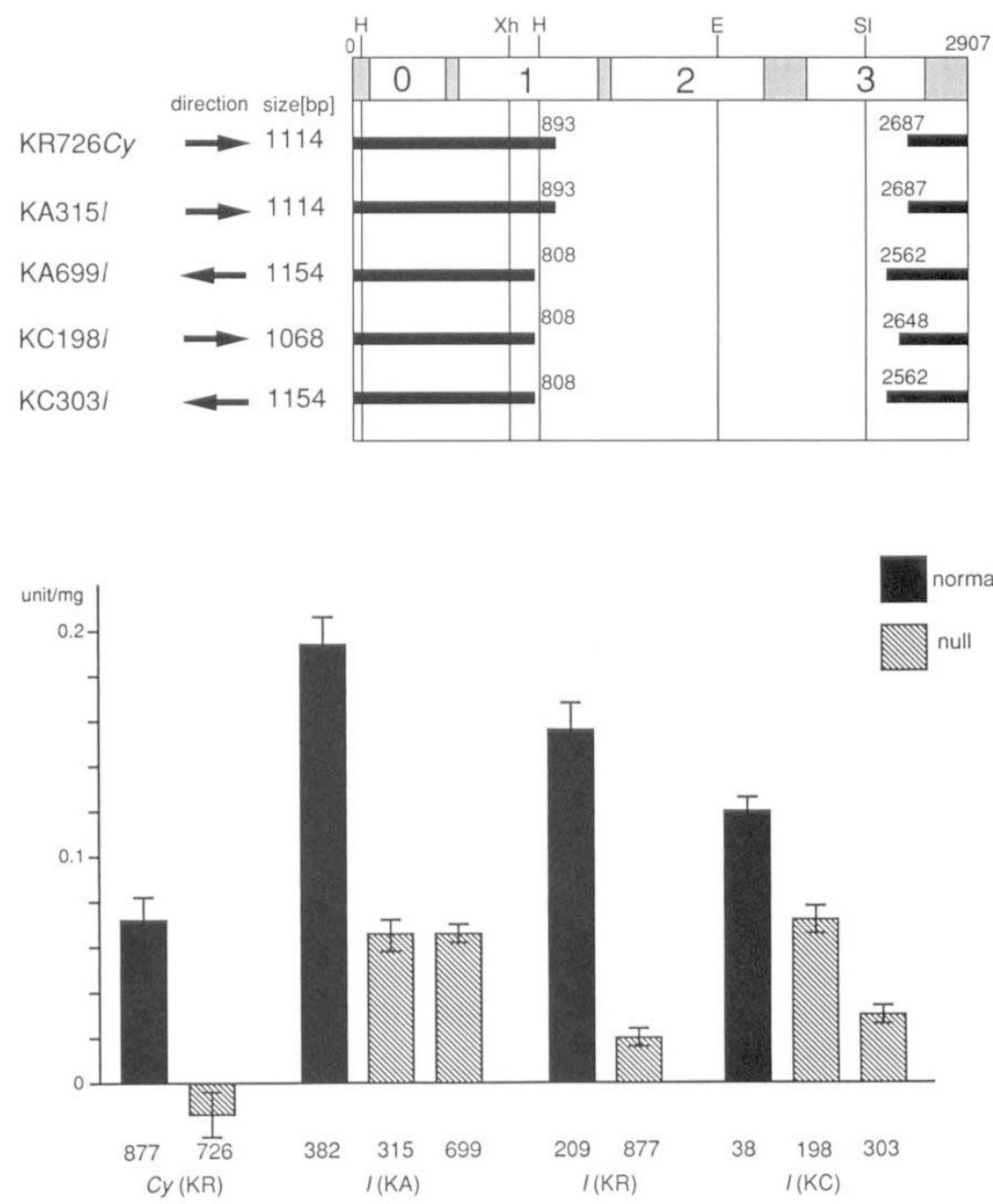

Fig. 5 Specific activity of $\alpha$ GPDH. The mean $\alpha$ GPDH specific activities of the null and control lines are shown with their 95 per cent confidence intervals. are large variations in the enzyme activity among the control lines. There is known genetic variation in the enzyme activities in flies from natural populations (Laurie-Ahlberg, 1985) and the differences in the control lines reflect the variation in the stem chromosomes sampled from natural populations. Comparing the relative activities in the null lines there is an apparent tendency that the closer the $P$ element insertions are to the initiation codon, the greater the reduction in $\alpha \mathrm{GPDH}$ activity. The orientation of the $P$ element did not affect the activity level (e.g. KA315l and KA699 $l$ have $P$ element insertions in opposite directions but show the same enzyme activity level).

\section{Discussion}

The mutation accumulation experiment was conducted to obtain reliable mutation rates under conditions in which the effects of the transposons are reduced in two ways. One is using old laboratory strains and the other is using a native background to minimize the hybrid dysgenesis (Kidwell et al., 1977). In spite of our effort, five of the six Gpdh null mutants studied in this experiment had insertions of internally deleted $P$ elements. $P$ element activity in these mutation accumulation lines was examined by the $s n^{\mathrm{w}}$ hypermutability test (Engles \& Preston, 1979, 1980). Twelve lines from the KA series, five lines from the KR series and 13 lines from the $\mathrm{KC}$ series were randomly sampled and examined. In total, 744, 152 and 2439 flies were counted in the $\mathrm{KA}, \mathrm{KR}$ and $\mathrm{KC}$ series, respectively. Only two mutants $\left(s n^{\mathrm{e}}\right.$ in the $\mathrm{KC}$ series) were found. This finding suggests that the $P$ element activity is very low in these lines, but suppression was not complete and did allow $P$ element transposition at a low level.

It has been suggested that $P$ element insertions often lie near the transcription start sites of genes, especially the genes that are active in germ lines (Tsubota et al., 1985; Kelly et al., 1987). It has been shown that $P$ 
element insertions occurred between the TATA box and the transcription initiation site of the Gpdh null mutations obtained in natural populations (Reed \& Gibson, 1993). All the insertions occurred in precisely the same nucleotide position and this is the same for the insertions of KA315l and KA699 $l$ in our experiment. The nucleotide sequence at this site was $5^{\prime}$ GTGCAAAC-3'. This site does resemble the reported consensus (O'Hare \& Rubin, 1983), but not completely. A similar observation was reported for the $s n$ (singed) locus (Roiha et al., 1988). The fact that all the $P$ element insertions occur in such a small region (probably within $169 \mathrm{bp}$ ) in both natural populations and the mutation accumulation lines indicates that this region is a hotspot of the $G p d h$ gene. Changes in both site specificity and chromatin structure during transcription may provide the preference for the insertion (Eissenberg \& Elgin, 1987; Roiha et al., 1988).

There is a large variation in the enzyme activity among insertional null mutations. There is also a clear tendency that the nearer the insertion is to the transcription initiation site the larger the reduction in the activity becomes. The activity level is most likely reflecting the transcriptional level of $\alpha \mathrm{GPDH}$ mRNA (Reed \& Gibson, 1993). It is not known whether other $P$ element insertions occurred upstream of this region in the mutation accumulation lines; they might not have been detected because their enzyme activities were little affected.

The deletion mutant (KR877l) lacked the region that contains the seventh and eighth exons. These regions are necessary for the production of GPDH-1 and GPDH-2 (Bewley et al., 1989) (Fig. 1). The trace amount of the enzyme activity ( 12 per cent of the control line) may result from the production of GPDH-3 that is made up of exon 1 to exon 6 (Fig. 1) and is mainly produced in larvae. It has been shown that excision of the $P$ element often accompanies deletions in the range beyond $6 \mathrm{~kb}$ (Salz et al., 1987). The events that include $P$ element insertion and subsequent excision in this region are suggested to be one of the causes for this deletion.

The null mutation rate of the Gpdh locus in the previous experiment (Mukai \& Cockerham, 1977) was $2.31 \times 10^{-5}(=7 / 302720)$ per generation and was not significantly higher than the other four loci (Adh: $2 / 307220=6.51 \times 10^{-6}, M d h: 6 / 309550=$ $1.94 \times 10^{-5}$, Hex-C: $2 / 348198=5.74 \times 10^{-6}$ and Amy: 0/234 610 =0.0), and it was also found that their lines did not have any $P$ element (Harada et al., 1990). The null mutation rate of the Gpdh locus in the present experiment was $1.04 \times 10^{-4}=25 / 239533$ per generation (Harada et al., 1993). This is significantly higher than the other loci $\left(A d h: 6 / 240669=2.49 \times 10^{-5}\right.$, Mdh: $4 / 237862=1.68 \times 10^{-5}$, Hex-C: $2 / 239418=$
$8.35 \times 10^{-6}, \quad$ Got: $3 / 241 \quad 121=1.24 \times 10^{-5}, \quad$ Dip: $3 / 240370=1.25 \times 10^{-5}$ ). The mutation rates in $A d h$, $M d h$ and $H e x-C$ were not significantly different from that of the previous estimations. $P$ element transposition is thus suggested to be the major cause of the higher null mutation rate at the Gpdh locus in the present experiment. No significant differences in the mutation rates at the $A d h, M d h$ and $H e x-C$ loci between the present and the previous experiments suggests that these loci tend not to be affected by the $P$ element insertion. Our observation is also applicable to natural populations. Voelker et al. (1980a) examined the null allele frequencies at 25 allozyme loci in a Raleigh, North Carolina population. The same loci were examined by Langley et al. (1981) in a London, UK population. Although there was significant heterogeneity among the loci in null frequencies, these two populations appeared to have the same frequencies at each locus. This suggests that the null alleles in these loci are in a mutation-selection balance and thus suggests that the higher null allele frequency reflects the higher mutation rate. The $G p d h$ null allele frequencies in these populations were significantly higher than the other loci linked on the second chromosome (i.e $A d h, M d h$, Got, Dip and Hex-C). It has been suggested that considerably large genetic loads concerning viability, which were observed in the Raleigh population in 1970 (Mukai \& Yamaguchi, 1974), were caused by the rapid invasion of the $P$ element (Harada et al., 1991). It is thus probable that the high null frequency at the Gpdh locus in the Raleigh population is caused by the insertion of $P$ elements. This may also be true for the London population. In fact, one of the Gpdh null mutations found in the London population had an insertion of the defective $P$ element (Reed \& Gibson, 1993).

Our experiment showed that the $P$ elements affect the Gpdh null mutation rate. Can the other transposable elements cause null mutations in enzyme loci? The molecular structures of the Adh null alleles from natural populations have been previously reported (Gibson \& Wilks, 1989; Gibson et al., 1992). One of them contained a stop codon whereas some of them had insertions or deletions; however, there was no example which clearly showed that transposons caused null mutations. Their data suggest that the Adh locus tends not to be inserted by the $P$ or other elements. Our mutation accumulation lines also harboured hobo and $l$ elements which are also known to be agents of hybrid dysgenesis. Because no hobo or $l$ element insertions were found in the Gpdh null mutations, they do not seem to have affected the null mutation rates at this locus. However, their effect on the other loci is not known. A further analysis of the molecular structure of null mutations in various loci is thus still necessary 
before we can draw any general conclusions as to the cause of null mutations.

\section{Acknowledgements}

We thank H. Tachida and M. M. Green for many helpful comments on the manuscript. This work was supported by a Grant-in-Aid for Scientific Research from the Ministry of Education, Science and Culture, Japan.

\section{References}

BEWLEY, G. C., COOK, J. L., KUSAKABE, S., MUKAI, T., RIGBY, D. L. AND CHAMBERS, G. K. 1989. Sequence, structure and evolution of the gene coding for sn-glycerol-3-phosphate dehydrogenase in Drosophila melanogaster. Nucl. Acids Res., 17, 8553-8567.

BLACK, D. M., JACKSON, M. S., KIDWELL, M. G. AND DOVER, G. A. 1987. KP elements repress $\mathrm{P}$-induced hybrid dysgenesis in Drosophila melanogaster. Eur. Mol. Biol. Organ. J., 6, 4125-4135.

DALY, K. AND CLARKE, B. 1981. Selection associated with the alcohol dehydrogenase locus in Drosophila melanogaster: differential survival of adults maintained on low concentrations of ethanol. Heredity, 46, 219-226.

EISSENBERG, J. C. AND Elgin, S. C. R. 1987. Hsp $28^{\text {st }}$; a P-element insertion mutation that alters the expression of a heat shock gene in Drosophila melanogaster. Genetics, 115, 333-340.

ENGELS, w. R. AND PRESTON, C. R. 1979. Hybrid dysgenesis in Drosophila melanogaster: the biology of female and male sterility. Genetics, 92, 161-174.

ENGELS, W. R. AND PRESTON, C. R. 1980. Components of hybrid dysgenesis in a wild population of Drosophila melanogaster. Genetics, 95, 111-128.

GIBSON, J. B. AND wILKS, A. v. 1989. Molecular structure of a naturally occurring alcohol dehydrogenase null activity allele in Drosophila melanogaster. Biochem. Genet., 27, 679-688.

GIBSON, J. B., WILKS, A. V. AND AGROTIS, A. 1992. Molecular relationships between alcohol dehydrogenase null-activity alleles from natural populations of Drosophila melanogaster. Mol. Biol. Evol., 9, 250-260.

HARADA, K., KUSAKABE, S. AND MUKAI, T. 1991. Comparative study of $P$ element activity in two natural populations of Drosophila melanogaster. Jpn. J. Genet., 66, 725-737.

HARADA, K., KUSAKABE, S., YAMAZAKI, T. AND MUKAI, T. 1993. Spontaneous mutation rates in null and band-morph mutations of enzyme loci in Drosophila melanogaster. Jpn. J. Genet., 68, 605-616.

HARADA, K., YUKUHIRO, K. AND MUKAI, T. 1990. Transposition rates of movable genetic elements in Drosophila melanogastera. Proc. Natl. Acad. Sci. U.S.A., 87, 3248-3252.

KELLY, M. R., KIDD, S., BERG, R. L. AND YOUNG, M. W. 1987. Restriction of $P$-element insertions at the notch locus of Drosophila melanogaster. Mol. Cell. Biol., 7, 1545-1548.
KIDWELL, M. G., KIDWELL, J. F. AND SVED, J. A. 1977. Hybrid dysgenesis in Drosophila melanogaster: a syndrome of aberrant traits including mutation, sterility and male recombination. Genetics, 86, 813-833.

KogA, A., KuSAKABE, S., TAJimA, F., HARADA, K., BEWLEY, C. AND MUKAI, T. 1988. Wide-spread polymorphism of a tandem duplication in the region of the glycerol-3-phosphate dehydrogenase gene in Drosophila melanogaster. Proc. Jpn. Acad., 64, 9-12.

LANGLEY, C. H., VOELKER, R. A., LEIGH BROWN, A. J., OHNISHI, S., DICKSON, B. AND MONTOGOMERY, E. 1981. Null allele frequencies at allozyme loci in natural populations of Drosophila melanogaster. Genetics, 99, 151-156.

LAURIE-AHLBERG, C. C. 1985 . Genetic variation affecting the expression of enzyme-coding genes in Drosophila: an evolutionary perspective. In: Rattazzi, M. C., Scandalios, J. G. and Whitt, G. S. (eds) Isozymes: Current Topics in Biology and Medical Research, pp. 33-88. IRL Press, Oxford.

LINDSEY, D. L. AND ZIMM, G. G. 1992. The Genome of Drosophila melanogaster. Academic Press, New York.

LOWRY, O. H., ROSEBROUGH, M. N. J., FARR, A. L. AND RANDALL, R. J. 1951. Protein measurement with the folin phenol reagent. J. Biol. Chem., 193, 265-275.

MUKAI, T. AND COCKERHAM, C. C. 1977. Spontaneous mutation rates at enzyme loci in Drosophila melanogaster. Proc. Natl. Acad. Sci. U.S.A., 74, 2514-2517.

MUKAI, T. AND YAMAGUCHI, o. 1974. The genetic structure of natural populations of Drosophila melanogaster. XI. Genetic variability in a local population. Genetics, 76, 339-366.

O'HARE, K., DRIVER, A., MCGRATH, S. AND JOHONSON-SCHILTZ, D. M. 1992. Distribution and structure of cloned P elements from the Drosophila melanogaster P strain $\pi_{2}$. Genet. Res., 60, 33-41.

O'HARE, K. AND RUBIN, G. M. 1983. Structures of P transposable elements and their sites of insertion and excision in the Drosophila melanogaster genome. Cell, 34, 25-35.

REED, D. S. AND GIBSON, J. B. 1993. Defective P element insertions affect the expression of $s n$-glycerol-3-phosphate dehydrogenase alleles in natural populations of Drosophila melanogaster. Proc. R. Soc. B, 251, 39-45.

RoIHA, H., RUBIN, G. M. AND O'HARE, K. 1988. $P$ element insertions and rearrangements at the singed locus of Drosophila melanogaster. Genetics, 119, 75-83.

SALZ, H. K., CLINE, T. W. AND SCHEDL, P. 1987. Functional changes associated with structural alterations induced by mobilization of a $P$ element inserted in the Sex-lethal gene of Drosophila. Genetics, 117, 221-231.

SAMBROOK, J., FITSCH, E. F. AND MANIATIS, T. 1989. Molecular Cloning, a Laboratory Manual, 2nd edn. Cold Spring Harbor Laboratory, Cold Spring Harbor.

SANGER, F., NICKLEN, S. AND COULSON, A. R. 1977. DNA sequencing with chain-terminating inhibitors. Proc. Natl. Acad. Sci. U.S.A., 74, 5463-5467.

TAKANO, T. S., KUSAKABE, S., KogA, S. AND MUKAI, T. 1989. Polymorphism of the number of tandemly multiplicated glycerol-3-phosphate dehydrogenase genes in Drosophila melanogaster. Proc. Natl. Acad. Sci. U.S.A., 86, 5000-5004. 
TSUBOTA, S., ASHBURNER, M. AND SCHEDL, P. 1985. P-elementinduced control mutations at the $r$ gene of Drosophila melanogaster. Mol. Cell. Biol., 5, 2567-2574.

VOELKER. R. A., LANGLEY, C. H., LEIGH-BROWN, A. J., OHNISHI, S., DOCKSON, B., MONTGOMERY, E. AND SMITH, S. C. 1980a. Enzyme null alleles in natural populations of Drosophila melanogaster. Frequencies in a North Carolina population. Proc. Natl. Acad. Sci. U.S.A., 77, 1091-1095.
VoelKer, R. A., SChAfFER, H. E. AND MUKAI, T. 1980b. Spontaneous allozyme mutations in Drosophila melanogaster. rate of occurrence and nature of the mutants. Genetics, 94, 961-968.

YAMAGUCHI, O. AND MUKAI, T. 1976. High rates of occurrence of spontaneous chromosome aberrations in Drosophila melanogaster. Genetics, 83, 409-422. 